\title{
Investigating Role of Ferritin in Ex Vivo Erythropoiesis by Block-face SEM and STEM-EELS
}

Maria Aronova ${ }^{1}$, Seung-Jae Noh ${ }^{2}$, Guofeng Zhang ${ }^{1}$, Colleen Byrnes ${ }^{3}$, Emily Riehm Meier ${ }^{4}$, Young Kim ${ }^{5}$ and Richard Leapman ${ }^{1}$

${ }^{1}$ NIBIB, National Institutes of Health, Bethesda, Maryland, United States, ${ }^{2}$ Penta Medix Co., United States, ${ }^{3}$ NIDDK, National Institutes of Health, United States, ${ }^{4}$ Indiana Hemophilia \& Thrombosis Center, United States, ${ }^{5}$ US Naval Research Laboratory, United States

Mammalian erythropoiesis is a precisely orchestrated process, whereby erythroid progenitor cells terminally differentiate to red blood cells (RBCs) in vivo, or to reticulocytes ex vivo. However, the path of the labile iron pool and its transport to mitochondria for heme production is not yet fully understood (1). Existing models for erythropoiesis do not involve a central role for the ubiquitous iron storage protein ferritin. Instead, it is believed that incoming endosomal Fe3+ attached to transferrin is first reduced to $\mathrm{Fe} 2+$ before entering the cytoplasm through divalent metal ion transporters, and is then immediately taken up into mitochondria through the mitoferrin-1 transporter. Erythroblast differentiation ex vivo mimics the in vivo process, in which progenitor cells accumulate hemoglobin, eventually losing their nuclei and other organelles to form RBCs that fulfill their primary function of oxygen transport through the vascular system.

To investigate further the accumulation of iron during erythropoiesis, we have analyzed the ultrastructural and compositional changes that occur in human CD34+ stem cells grown in ex vivo culture as an erythroblast sub-lineage differentiates over a three-week period into reticulocytes (2). We have applied electron energy loss spectroscopy (EELS) in the scanning transmission electron microscope (STEM) using a Thermo Fisher/FEI Tecnai TF30 TEM equipped with a Gatan Quantum EELS system to image iron in subcellular organelles of these differentiating cells. Samples were prepared conventionally with osmium fixative and plastic embedding but without other heavy atom staining. Dark-field STEM images from 100$\mathrm{nm}$ thick sections revealed ultrastructural changes at day 7 , when mitochondria and membrane-bound lysosomes gather at the Golgi pole. Within compartments of lysosomes, nanoparticles approximately 10 $\mathrm{nm}$ in diameter, visible as bright punctate features in dark-field images, were indicative of ferritin (3-5). STEM-EELS maps around the Fe L2,3 edge resonance at $\sim 710 \mathrm{eV}$, confirmed the presence of Fe $3+$ iron in each nanoparticle. Quantitative EELS analysis of 96 such iron-containing particles gave a mean $\mathrm{Fe}$ content of 2,400 \pm 800 atoms, again consistent with the known iron content of individual ferritin cores. By counting numbers of particles per unit area in STEM images from lysosomes and knowing the section thickness $(\sim 100 \mathrm{~nm})$, it was possible to determine the number of ferritin cores, and therefore the number of iron atoms per unit volume of lysosomes.

We have combined these iron measurements with 3D ultrastructural measurements obtained by serial block-face scanning electron microscopy (SBF-SEM) using a Zeiss Sigma SEM equipped with a Gatan 3View serial block-face imaging system (6); samples for SBF-SEM were prepared with additional heavy atom staining that was omitted in the STEM-EELS experiments. By determining the total volumes occupied by the ferritin-containing lysosomes, it was possible to estimate the total content of lysosomal iron per cell during the different stages of differentiation. The 3D SBF-SEM images also provided important information about proximity of the lysosomes to mitochondria as well as the overall rearrangements of ultrastructure during erythropoiesis. It was found that at day 7 of ex vivo 
erythropoiesis, there were approximately $2 \times 108$ iron atoms per cell, i.e., $20 \%$ of the number of iron atoms in a mature $\mathrm{RBC}$ or reticulocyte.

Our results support the hypothesis that lysosomal ferritin iron depots are utilized by developing reticulocytes to continue heme production after much of the cellular machinery has been expelled (7).
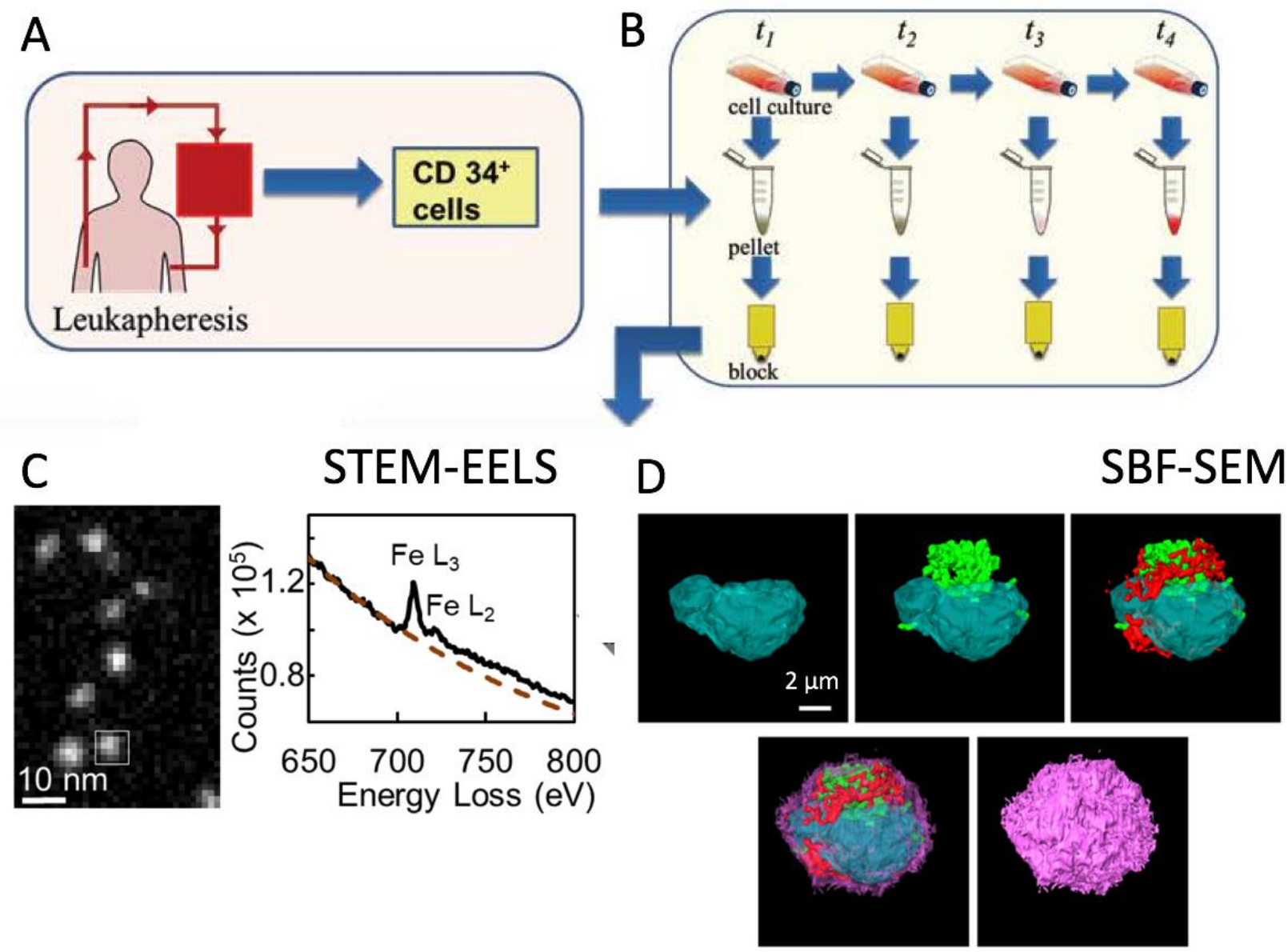

Figure 1. (A) CD34+ stem cells are isolated from healthy volunteers, via leukapheresis, and cultured in serum-free media. (B) At four different time points of differentiation: $\mathrm{t} 1=$ day 0 , $\mathrm{t} 2=$ day 7 , $\mathrm{t} 3=$ day 14 (7 days after erythropoietin exposure), t4 = day 19-21 (14 days after erythropoietin exposure), cells are collected and pelleted before undergoing fixation, staining and embedding. (C) STEM and EELS analysis of erythroid sub-lineage reveals a high concentration of ferritin molecules contained in lysosomes between days 7 and 14. (D) SBF-SEM images of differentiating erythroblast at day 7 showing nucleus (blue), clustered lysosomes (green), surrounding mitochondria (red), and ruffled plasma membrane (pink).

\section{References}


1. M.U. Muckenthaler et al. Cell 168(3), 344-361 (2017).

2. M.A. Aronova et al., bioRxiv 2021. doi: https://doi.org/10.1101/866889

3. R.D. Leapman. J. Microsc. 210, 5-15 (2003).

4. M. Fukunaga et al. Proc. Natl. Acad. Sci. USA, 107(8), 3834-3839 (2010).

5. P. Zhang et al. J. Struct. Biol. 150(2), 144-153 (2005).

6. W. Denk, H. Horstmann. Plos Biol. 2(11), 1900-1909 (2004).

7. Research supported by the intramural programs of NIBIB and NIDDK, NIH, and the Office of Naval Research, USNRL. The authors thank Dr. J. L. Miller for helpful discussions. 